\title{
Renal Replacement Lipomatosis-A Rare Benign Condition
}

\author{
Vishal Kadeli1,2, Rajendra Nerli ${ }^{1,2}{ }^{*}$, Shridhar Ghagane ${ }^{3}$, Murigendra B. Hiremath ${ }^{4}$, Neeraj Dixit ${ }^{3}$ \\ ${ }^{1}$ Department of Urology, JN Medical College, KLE Academy of Higher Education \& Research (Deemed-to-Be-University), \\ JNMC Campus, Belagavi, India \\ ${ }^{2}$ KLES Kidney Foundation, KLES Dr. Prabhakar Kore Hospital \& M.R.C., Belagavi, India \\ ${ }^{3}$ Department of Urology, KLES Kidney Foundation, KLES Dr. Prabhakar Kore Hospital \& M.R.C., Belagavi, India \\ ${ }^{4}$ Department of Biotechnology and Microbiology, Karnatak University, Dharwad, India \\ Email: *rbnerli@gmail.com
}

How to cite this paper: Kadeli, V., Nerli, R., Ghagane, S., Hiremath, M.B. and Dixit, N. (2018) Renal Replacement Lipomatosis-A Rare Benign Condition. Surgical Science, 9, 306-310.

https://doi.org/10.4236/ss.2018.99037

Received: March 1, 2018

Accepted: September 24, 2018

Published: September 27, 2018

Copyright (c) 2018 by authors and Scientific Research Publishing Inc. This work is licensed under the Creative Commons Attribution-NonCommercial International License (CC BY-NC 4.0).

http://creativecommons.org/licenses/by-nc/4.0/

\begin{abstract}
Introduction: Renal replacement lipomatosis is a rare condition that occurs at the end of the spectrum of renal tissue replacement by fat. It is usually unilateral and occurs as a result of severe renal atrophy or destruction usually due to chronic calculus disease, chronic pyelonephritis and renal tuberculosis. The most commonly used diagnostic investigation remains CECT (contrast enhanced computed tomography) scan. Herein we report our series of patients with total renal replacement lipomatosistreated by nephrectomy. Materials \& Methods: We retrospectively reviewed our hospital data for cases of RRL, presented and treated during the period Jan. 2006-Dec. 2015. The age, gender, presenting symptoms, clinical and laboratory findings were noted. The indications for surgery, postoperative outcomes and complications were similarly noted and analyzed. Results: Five patients (three females and two males) with a mean age of $48.4 \pm 3.2$ years presented with symptoms of pain. Computed tomography (CT) in these patients showed the affected kidney to be completely distorted and replaced by ill-defined fatty attenuating lesion. Open nephrectomy was done in all these patients. Histo-pathological report (HPR) revealed features of RRL with renal calculi. Conclusions: Renal replacement lipomatosis is an uncommon entity, and one should have a high index of suspicion when dealing with suspicious lesions similar to xanthogranulomatous pyelonephritis. Specific imaging, operative, and pathological differences may provide clues for the differential diagnosis.
\end{abstract}

\section{Keywords}

Renal Replacement Lipomatosis, Nephrectomy, Pathogenesis 


\section{Introduction}

Renal replacement lipomatosis (RRL) is a relatively uncommon disease entity, and is commonly mistaken for xanthogranulomatous pyelonephritis (XGP), which may be due to lack of awareness among urologists, radiologists, and pathologists [1] [2]. RRL, was first reported by Brown in 1861 [2] [3]. RRL, renal sinus lipomatosis and fibro-lipomatosis represent the various stages of the same disease, wherein the normal renal sinus fat and the peri-renal fat gradually increase to replace the renal parenchyma. Renal sinus lipomatosis which is the mildest form, is usually associated with obesity, renal atrophy (secondary to aging, atherosclerosis), Cushing's syndrome or the use of exogenous steroids [2] [4] [5]. This mild form rarely produces symptoms because of the absence of calyceal obstruction [4], and is a common finding at autopsy [2]. In RRL which is at the other end of the spectrum, the entire renal parenchyma is replaced by adipose tissue, usually secondary to calculous disease and long standing inflammatory/infectious process. The proliferation of the fat cells into the interstitium of the kidney is mediated by the connective tissue [2] [3].

Xanthogranulomatous pyelonephritis is an uncommon renal inflammatory disease characterized by the destruction and replacement of normal parenchyma by sheets of lipid-laden histiocytes, resulting in enlarged renal parenchyma. XGP begins within the pelvis and calyces and subsequently extends into and destroys renal parenchymal and adjacent tissues. All theories agree that the primary factors involved in the development of XGP are bacterial infection, obstruction and calculous disease. Other possible interrelated factors include venous occlusion and hemorrhage, abnormal lipid metabolism, lymphatic blockage, failure of antimicrobial therapy, altered immunologic competence, and renal ischemia [2] [6]. We retrospectively reviewed our hospital data and reported our series of patients diagnosed to have had RRL.

\section{Materials and Methods}

With the consent obtained from the institutional ethics committee, we retrospectively reviewed the KLES Hospital data for patients undergoing nephrectomy. Inclusion criteria included all cases of RRL that presented to the hospital and treated during the study period Jan. 2006-Dec. 2015. The age, gender, presenting symptoms, clinical and laboratory findings were noted. The indications for surgery, postoperative outcomes and complications were similarly noted and analyzed.

\section{Results}

During the study period there were five patients (three females and two males) that fulfilled the criteria of RRL. The mean age at presentation was $48.4 \pm 3.2$ years. Pain in abdomen, discomfort and vague abdominal mass were the primary presenting symptoms (Table 1$)$. The symptoms were of long standing $(>12$ months). Plain $\mathrm{x}$-rays revealed renal stones in all the patients. Computed 
Table 1. Details of patients with RRL.

\begin{tabular}{cccccc}
\hline No. & Age/Gender & Symptoms & Side & Co-morbidity & Outcome \\
\hline 1 & $43 / \mathrm{F}$ & Pain, hematuria & $\mathrm{R}$ & Renal stone/DM & Nephrectomy \\
2 & $52 / \mathrm{M}$ & Pain, mass & $\mathrm{R}$ & Renal stone & -do- \\
3 & $47 / \mathrm{F}$ & Pain, mass & $\mathrm{L}$ & Renal stone/DM & -do- \\
4 & $51 / \mathrm{F}$ & Pain, discomfort, mass & $\mathrm{L}$ & Renal stone & -do- \\
5 & $49 / \mathrm{M}$ & Pain, discomfort & $\mathrm{R}$ & Renal stone/HTN & -do- \\
\hline
\end{tabular}

tomography (CT) in these patients showed the morphology of the affected kidney to be completely distorted and replaced by ill-defined fatty attenuating lesion, with irregular heterogeneous enhancing lesions representing inflammatory changes in the perinephric fat. The radionuclide studies revealed very poorly/non-functioning kidneys. Open Nephrectomy was done in all these patients. The indications for nephrectomy in all these patients were a poorly/non-functioning symptomatic kidney.

Macroscopic examination of the nephrectomy specimen showed an irregular, friable, green/yellow mass replacing the entire renal parenchyma, with a pale and shiny mucosa filled with fine granular calculi. The fatty tissue inside the renal parenchyma was paler than the perirrenal fat, differentiating xanthogranulomatous pyelonephritis from renal replacement lipomatosis. Microscopic examination revealed abundance of chronic inflammatory cell infiltrate with few, isolated, scattered glomeruli and encroachment by adipocytes.

The post-operative period in all the five patients who underwent nephrectomy for RRL was uneventful. The drain and the urethral catheter was removed after 48 hours. All patients took oral feeds within 24 hours following surgery and were discharged from the hospital within 96 hours. Post-operative serum creatinine stabilized to around $1.2 \pm 0.325 \mathrm{mg} \%$. The mean follow-up period was 14 months and all patients were doing fine.

During the same study period, six other patients (five females and one male) with a mean age of $54.2 \pm 5.7$ years underwent nephrectomy for poorly functioning, infected kidneys with histopathological examination revealing xanthogranulomatous pyelonephritis. All these six patients were diabetic with elevated HbA1c (Table 2). Two of these six patients had post-operative fever, which was treated by antipyretics. On patient developed wound infection, which needed wound dressing. The mean post-operative serum creatinine was $1.85 \pm 1.55 \mathrm{mg} \%$.

\section{Discussion}

Renal replacement lipomatosis is an aggressive form of renal sinus lipomatosis. It is usually an unilateral process accompanied by parenchymal atrophy that results from calculousdisease, chronic hydronephrosis, or infection [2] [7]. Often there is extension of lipomatosis into the perirenal and periureteral spaces as 
Table 2. RRL in comparison with xanthogranulomatous pyelonephritis.

\begin{tabular}{ccc}
\hline Parameters & RRL & XGP \\
\hline Age & $48.4 \pm 3.2$ & $54.2 \pm 5.7$ \\
Diabetes Mellitus & $40 \%$ & $100 \%$ \\
Urine culture & Negative in all & E. Coli \\
Renal stone & $100 \%$ & $50 \%$ \\
HPR & $\begin{array}{c}\text { Atrophic renal parenchyma } \\
\text { replaced by fatty tissue } \\
\text { proliferation }\end{array}$ & $\begin{array}{c}\text { Xanthoma cells infiltrate and substitute } \\
\text { necrotic renal tissue resulting in a } \\
\text { lipomatous degeneration }\end{array}$ \\
\hline
\end{tabular}

well. RRL usually occurs between the fifth and seventh decade of life. The patient may be asymptomatic or may present with a varied clinical picture, the most common manifestations being urinary tract infections, flank pain, weight loss, hematuria, fever, and palpable mass.

The major differential diagnosis for RRL of the kidney include malakoplakia; fat containing tumors such as angiomyolipomas, lipomas, and liposarcomas; XGP, particularly in the presence of calculi and inflammatory changes ; and transitional cell carcinoma of the renal sinus [2]. Sonography is a suitable modality for preliminary evaluation of patients with RRL. The salient findings include an enlarged echogenic central sinus, containing a calculus in nearly $70 \%$ of the cases [2] [8]. The central distribution of adipose tissue in the renal sinus is considered characteristic of RRL [4]. Computed tomography can be used to further characterize the disease and is better than sonography in delineating perirenal changes. The fat in renal replacement lipomatosis is concentrated in the renal sinus and hilum, with a diffuse extension perirenally. Recently MR (magnetic resonance) and MR urography with gadolinium contrast have come up as the most accurate diagnostic tools for replacement lipomatosis showing the presence of perirenal fat intensity signal, obstruction, dilated ureter, level of obstruction, and atrophic kidney [4] [9]. Invariably the kidney is non-functional in these cases of RRL and nephrectomy would appear the treatment of choice.

Romero et al. [2] reported on a 63 year old female and a 51 year old obese female, who presented with flank pain and urinary tract infection. Computed tomography (CT) scan showed an enlarged right kidney with hydronephrosis, parenchymal atrophy, and calculi located in the renal pelvis, with marked fatty proliferation within the right renal sinus. These cases were initially misdiagnosed. The patients underwent a nephrectomy and HPR diagnosed them as RRL.

Due to the rarity of the disease, one may not have adequate experience to diagnose this entity. Most clinicians would make an initial diagnosis of a solid mass and tumor, and the other probable benign conditions would be ignored. Considering the longtime irritation from a urinary stone and the long disease history, some would even think of the possibility of squamous cell carcinoma (SCC) of the pelvis, although the CT findings would not be compatible with 
SCC. It is important for urologists to recognize this condition. As the condition is rare and the number in this study is small, one cannot make treatment guidelines or suggest diagnostic tips.

\section{Conclusion}

Renal replacement lipomatosis is an uncommon entity, and one should have a high index of suspicion when dealing with suspicious lesions similar to xanthogranulomatous pyelonephritis. Specific imaging, operative, and pathological differences may provide clues for the differential diagnosis.

\section{Conflicts of Interest}

The authors declare no conflicts of interest regarding the publication of this paper.

\section{References}

[1] Xu, Y., Liu, R.L., Zhang, Z.H., Zhao, W.M. and Yang, Q.C. (2006) Renal Replacement Lipomatosis. European Surgical Research, 38, 385-387.

https://doi.org/10.1159/000094534

[2] Romero, F.R., Pilati, R., Caboclo, M.F.S., Silva, A.P.G., Cravo, M.A. and Filho, T.B. (2011) Renal Replacement Lipomatosis and Xanthogranulomatous Pyelonephritis: Differential Diagnosis. Revista da Associação Médica Brasileira, 57, 262-265. https://doi.org/10.1590/S0104-42302011000300005

[3] Dukes, C.E. (1938) The Pathology of Renal Lipomatosis. Proceedings of the Royal Society of Medicute, 31, 1361-1364.

[4] Kocaoglu, M., Bozlar, U., Sanal, H.T. and Guvenc, I. (2007) Replacement Lipomatosis: CT and MRI Findings of a Rare Renal Mass. The British Journal of Radiology, 80, 287-289. https://doi.org/10.1259/bjr/86054374

[5] Yagci, C., Kosucu, P., Yorubulut, M. and Akyar, S. (1999) Renal Replacement Lipomatosis: Ultrasonography and Computed Tomography Findings. European Radiology, 9, 1599-1601. https://doi.org/10.1007/s003300050893

[6] Sharma, S., Jhobta, A., Goyal, D., Surya, M., Sumala and Negi, A. (2006) Ureteral Involvement in Xanthogranulomatous Pyelonephritis-Rare Manifestation . Indian Journal of Radiology, 16, 243-245. https://doi.org/10.4103/0971-3026.29101

[7] Karasick, S. and Wechsler, R.J. (2000) Case 23: Replacement Lipomatosis of the Kidney. Radiology, 215, 754-756.

https://doi.org/10.1148/radiology.215.3.r00jn41754

[8] Rha, S.E., Byun, J.Y., Jung, S.E., et al. (2004) The Renal Sinus: Pathologic Spectrum and Multimodality Imaging Approach. RadioGraphics, 24, S117-S131. https://doi.org/10.1148/rg.24si045503

[9] Aerts, P., Van Hoe, L., Bosmans, H., Oyen, R., Marchal, G. and Baert, A.L. (1996) Breath-Hold MR Urography Using the HASTE Technique. American Journal of Roentgenology, 166, 543-545. https://doi.org/10.2214/ajr.166.3.8623625 